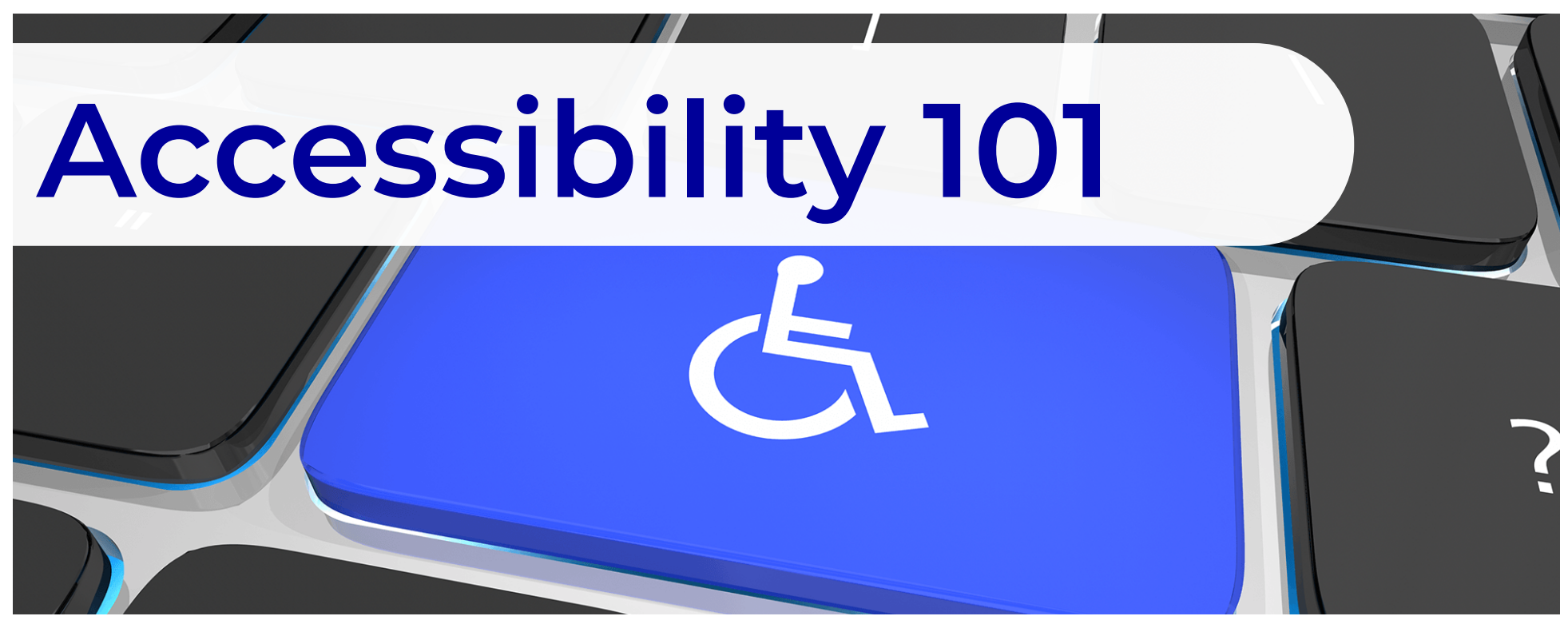

\title{
What is accessibility?
}

ACCESSIBILITY refers to whether a product or service is usable by all regardless of disability status. Principles of accessibility are not limited to physical spaces (such as stairs or curbs) - the same principles apply to websites and public facing documents. There are guidelines to follow to make documents, web pages, presentations, products, and research findings more accessible to people with disabilities.

\section{Why is it important?}

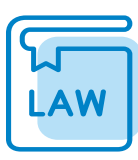

Law

Although not explicitly stated, accessible web content is often interpreted as a requirement of Title III of the Americans with Disabilities Act (ADA). Accessibility is also included in the Section 508 Standards.

These standards are mandated for Federal departments and agencies. ${ }^{2}$ This includes federally funded research.

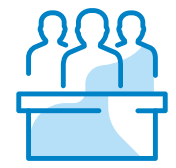

\section{Inclusion}

Many people with disabilities use assistive technology to access digital content or need content in alternative formats for it to be fully accessible to them.

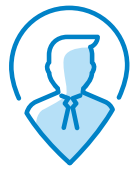

\section{Readership}

Making your research products accessible means your work can be read by more people. Accessibility

can also improve your visibility in search results. 


\section{What can I do about it?}

There are best practices to follow. Considerations will vary depending on the disability and/or need(s) that are being accommodated. The following are some of the most common accommodations and considerations to think about when preparing content for the public. These topics will be discussed at greater detail in our upcoming tip sheets, tutorials, and at our live sessions / webinars.

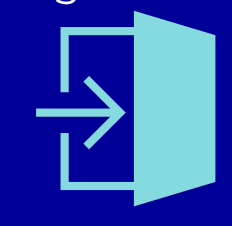

\section{Blind / Low Vision}

\section{Alternative text (alt-text)}

Images, including images of text, need to be described so that screen readers can interpret the content.

\section{Structure}

Screen readers need to know the structure of your document or webpage. Use built-in styles to tag paragraphs as headings or body text.

\section{heading 1 \\ paragraph \\ heading 2 \\ paragraph}

Screen readers cannot interpret visual cues. Bold text may indicate that the text is a heading when someone is reading it, but it means absolutely nothing for those using a screen reader. The screen reader needs to be cued that the text is a heading.

\section{Keyboard navigation}

Those who are blind cannot use a mouse to interact with their computers. They rely on keyboard navigation to find content.

- Users should be able to navigate throughout a webpage, form, or app using only their keyboard

\section{Audio descriptions $A D)$ )}

Audio descriptions (AD) are recommended for videos.

An audio description-also referred to as a video description, described video, or visual description-is an audio track used to describe the visual information in a video for a person who is blind or has low vision. 


\section{Captioning}

Provide captioning for videos. Communication Access Realtime Translation (CART) can be used for live events.

There are features in Zoom, Google Meets, Facebook, Microsoft

Teams, and YouTube that can add closed captioning and make your videos more accessible.

\section{Transcripts}

Provide transcripts for audio content. They can also be helpful for video content.

\section{ASL Interpreter}

There may be specific individual / group needs. For example, a group whose primary language is American Sign Language (ASL) may respond more positively to an ASL interpreter as opposed to closed captioning in English.

\section{1.) Colorblindness \& Contrast Issues}

\section{Colorblindness}

Do not use color as the only means of conveying information.

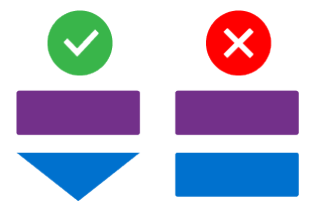

\section{Contrast ratio}

The contrast between the background and your foreground content must be sufficient so that the content is readable.

Contrast ratio is the measurement of difference between the background color and text. Guidance from the World Wide Web Consortium (W3C), the international web standards organization, states that text and images of text should contrast ratio of at least $4.5: 1 .^{3}$

\section{Useful tools for calculating your contrast ratio:}

- Colorcube color and contrast tool (https://oomphinc.github.io/colorcube)

- Color contrast checker* (https://www.levelaccess.com/color-contrast-checker)

\section{Colorblindness visualization tools:}

- Color blindness tool for images

(https://www.color-blindness.com/coblis-color-blindness-simulator)

- Chrome extension for websites 


\section{Plain language}

Use plain language whenever possible. Plain language means that the content you provide can be understood by your audience the first time them read it. It is also best to organize your content using the simplest structure possible. ${ }^{4}$

\section{Dyslexia}

People who are dyslexic have an easier time reading evenly spaced sans-serif fonts including-but not limited to-Arial, Verdana, Tahoma, and Century Gothic. A font size of $12-14$ points can also improve readability. 5,6

\section{Seizure warning}

Flashing colors, images, or lights can trigger seizures in some individuals ${ }^{5}$.

\section{Distractibility}

Some animations and GIFs (moving images) can be distracting for those with attention deficit hyperactivity disorder (ADHD).

\section{Helpful resources}

\section{(i) The Accessibility Cheat Sheet} (www.codlearningtech.org/2018/11/13/the-accessibility-cheat-sheet/)

(i) Digital A11y - a website devoted to resources on web accessibility (https://www.digitala17y.com/)

i The Ally project - a website devoted to web accessibility and, more broadly, digital accessibility (https://www.allyproject.com/)

(i) Alt-text decision tree (https://dev.w3.org/html5/alt-techniques/developer.html\#tree)

\section{References}

1. U.S. Department of Justice, Civil Rights Division, Disability Rights Section. A Guide to Disability Rights Laws. (2020, February). https://www. ada.gov/cguide.htm

2. U.S. Access Board. Rehabilitation Act of 1973. (n.d.) https://www.access-board.gov/law/ra.html\#text-of-section-508-of-the-rehabilitation-actof-1973-as-amended-29-usc-794d

3. W3C Recommendation. (2018, June 5). Web Content Accessibility Guidelines (WCAG) 2.1. https://www.w3.org/TR/WCAG21/\#contrastminimum

4. Universal Design Center, California State University Northridge. (n.d.) Accessibility (Ally) \& Universal Design. https://www.csun.edu/sites/ default/files/accessibility-ud-slides.pdf

5. Bureau of Internet Accessibility. (2019, February 12). How to Create Accessible Content and Designs for People with Dyslexia. https://www. boia.org/blog/how-to-create-accessible-content-and-designs-for-people-with-dyslexia

6. Dyslexic.com provided by Lansyst Ltd. (n.d) Quick Guide to Making Your Content Accessible. https://www.dyslexic.com/blog/quick-guidemaking-content-accessible/

\section{BOSTON UNIVERSITY}
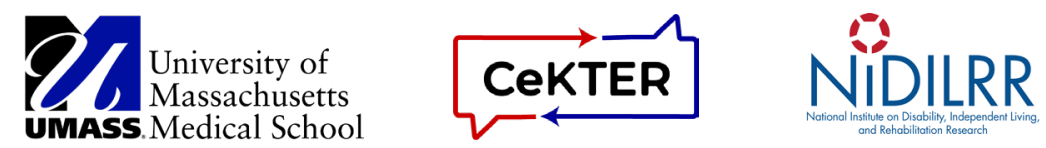

Suggested Citation: Murray, A. Accessibility 101: A Researcher's Guide to Making Content Accessible. Psychiatry Information in Brief 2021;18(2). 